\title{
Prevalence of Entamoeba Histolytica infection in patients with colitis (Ulcerative and Infective)
}

\author{
Luay I. Farhan Al-Rwi* \\ Maher M. Hassan Alakori* \\ Kifah H. Abdulghafour**
}

\author{
FICMS (Path) \\ FICMS (Ped) \\ MBChB, PhD (Path)
}

Abstract:

Fac Med Baghdad

2016; Vol.58, No.3

Received:June, 2016

Accepted:July.2016

Background: Ulcerative colitis (UC) is a chronic inflammatory disorder of the colon that often extends to involve the remainder of the large intestine in a continuous manner. Intestinal amoebiasis can present as a chronic, dysenteric syndrome of diarrhea, weight loss, and abdominal pain that can last for years and mimic ulcerative colitis.

Objective: Studying the prevalence of E..histolytica by using serological method ELISA for their detection, Investigate the possible role of E.histolytica as a cause of blood, diarrhea in non-ulcerative colitis group \&Comparison between serological detection of E.histolytica and stool examination.

Patients and Methods: Retrospective and prospective study was conducted on 48 patients (18 males, 30 females); 30 patients were diagnosed as ulcerative colitis and 18 as non ulcerative colitis patients by endoscopy, histopathology, radiology and laboratory examinations at Gastroenterology and Hepatology Teaching Hospital through the period between January 2008 and June 2008.

From all patients and control, a detailed medical history was taken; routine examination and relevant investigations were done. Blood and fresh stool specimens were collected from all patients and control. General stool examination and ELISA tests for IgG anti E.histolytica antibodies were assessed.

Results: Histological typing of ulcerative colitis grouped into chronic ulcerative colitis 42 cases $(86.67 \%)$ and active on chronic ulcerative colitis 6 cases (13.33\%). By general stool examination, E.histolytica was positive in (70\%) of ulcerative colitis $(53.33 \%$ cyst and $16.67 \%$ trophozoit) and results of ELISA test for IgG anti E.histolytica antibodies was significant compared with control group $(\mathrm{P}<0.05)$. General stool examination and IgG anti- E histolytica antibodies has been found that $75.8 \%$ of patients have positive IgG and cyst stage, and $77.8 \%$ were have positive $\mathrm{IgG}$ and trophozoit stage , while $24.2 \%$ of negative GSE results were also negative for IgG anti-E.histolytica antibodies, and the association was significant compared with control group $(\mathrm{P}<0.05)$.

Conclusion: E.histolytica IgG Abs were significantly higher in ulcerative colitis compared to non ulcerative colitis group .Close association between E.histolytica and ulcerative colitis also there is a high incidence of E.histolytica among group of non ulcerative colitis patients.

Sensitivity of detection of E.histolytica by ELISA and GSE is rather equal.

Key Words:,Entameobahistolytica, ulcerative colitis.

\section{Introduction:}

Ulcerative colitis it is a chronic inflammatory disorder of the colon that often extends to involve the remainder of the large intestine in a continuous manner. The disease is characterized by exacerbations and periods of remission. When the inflammation occurs in the rectum and lower part of the colon it is called ulcerative proctitis. If the entire colon is affected it is called pancolitis. If only the left side of the colon is affected it is called limited or distal colitis. $(1,2)$

It can be difficult to diagnose because its symptoms are similar to other intestinal disorders and to another type

*Pathology Dept.//College of Medicine/Baghdad University.

**Children Hospital.

kifahalani@yahoo.com of inflammatory bowel disease called Crohn's disease. (2) Ulcerative colitis can occur in people of any age, but it usually starts between the ages of 15 and 30, and less frequently between 50 and 70 years of age. It affects men and women equally and appears to run in families, with reports of up to 20 percent of people with ulcerative colitis having a family member or relative with ulcerative colitis or Crohn's disease. A higher incidence of ulcerative colitis is seen in White people. $(3,4)$

Amebiasis, which affects nearly 500 million people in the world, is more prevalent in developing countries in particular (5). It is difficult to distinguish IBD from colitis associated with amoeba according to both symptomatic and endoscopic appearance of the colon. It is not possible to 
establish a diagnosis by means of microscopic examination, because Sometimes IBD can co-exist with amebiasis. This, of course, leads to confusion in the diagnosis and treatment of the disease.(6) Intestinal amoebiasis, it's a cause of infective colitis can probably also present as a chronic, nondysenteric syndrome of diarrhea, weight loss, and abdominal pain that can last for years and mimic inflammatory bowel disease. Fulminant colitis with bowel necrosis leading to perforation and peritonitis occurs in only about $0.5 \%$ of cases, but it is associated with a mortality rate of more than $40 \%$. Patients with invasive amoebiasis living in developed countries generally acquire the infection in another country in which the pathogenic species is endemic. Areas that have high rates of amoebic infection include Asia, India, and Africa, Mexico and parts of Central and South America. Infection with pathogenic E. histolyticais is not a common cause of travelers' diarrhea, and gastrointestinal infection is uncommon in travelers who have spent less than one month in endemic areas. $(7,8)$

Despite the availability of sophisticated investigative procedures, differentiating invasive colonic amoebiasis from idiopathic inflammatory bowel disease (IBD) may be difficult. Presented to remind clinicians of the similarities in the clinical presentation and endoscopic features of these two conditions, and to highlight the difficulty in differentiating them. $(9,10)$

\section{Material and Methods:}

This case - series study included 48 patients (18 males and 30 females) 30 patients were diagnosed as ulcerative colitis their age ranged from 17 years to 60 years old with a mean age of ( 40.73 years \pm 12.269 ); and 18 patients as non-ulcerative colitis their age range from 3 years to 60 years old with the mean age $(27.46$ years \pm 22.423$)$. They were attendants of Gastroenterology and Hepatology Teaching Hospital in Baghdad from January 2008 to June 2008. The diagnosis in each case was established by clinical examination and simple investigation. They were subjected to colonoscopy or sigmoidoscopy after adequate preparation they were classified into 30 patients as ulcerative colitis and 18 patients as non ulcerative colitis. Eighteen healthy individuals ( 6 males and 12 females) were enrolled as a control in this study their age ranged from 18 to 48 years with a mean age of ( 31 years $\pm 9,883)$. Five $\mathrm{ml}$ venous blood was aspirated from and collected in dry plain tubes. Blood in plain tubes allowed to clot (for minimum 30 minutes) at room temperature $\left(25^{\circ} \mathrm{C}\right)$, then separated by centrifugation (3000 rpm for 5 minutes at $25^{\circ} \mathrm{C}$ ) to separate the serum and dispensed into sterile tightly closed Eppendrof tubes in $0.1 \mathrm{ml}$ aliquots and stored at $-20^{\circ} \mathrm{C}$ until assayed. Fresh stool from each patient and control was taken and kept in clean plastic container and directly examined after mount preparation by normal saline for E.histolytica trophozoit and iodine for E.histolytica cyst.

Material is enzyme immunoassay kit for detection of $\mathrm{IgG}$ antibodies to E. histolytica in serum (IVD Research INC quality diagnostic products).

\section{Result:}

This study included 48 patients attended Gastroenterology and Hepatology Teaching Hospital (a major referral center in Baghdad), and 18 healthy individual as control. Thirty cases $(45.45 \%)$ were ulcerative colitis, 18 (27.27\%) were non ulcerative colitis and 18 ( $27.27 \%$ as control .

There was a significant association between types of disease and endoscopic finding $(\mathrm{P}<0.05)$, it was found that about $60 \%$ of patients with ulcerative colitis had proctitis, $20 \%$ left side colitis, and 20\% pancolitis while finding were approximately equally distributed in patients with non ulcerative colitispatients between proctitis, left side colitis and normal (Table 1).

Table (1): distribution of the study groups according to the endoscopic findings.

\begin{tabular}{|c|c|c|c|c|c|c|}
\hline $\begin{array}{l}\text { Ulcerative } \\
\text { Colitis No }\end{array}$ & $\%$ & $\begin{array}{l}\text { Non } \\
\text { Ulcerative } \\
\text { Colitis No }\end{array}$ & $\%$ & $\begin{array}{c}\text { Total } \\
\text { number }\end{array}$ & $\%$ & $P$ value \\
\hline 18 & 60.0 & 7 & 38.9 & 25 & 52.1 & \multirow{5}{*}{0.003} \\
\hline 6 & 20.0 & 6 & 33.3 & 12 & 25.0 & \\
\hline 6 & 20.0 & $\mathbf{0}$ & 0.0 & 6 & 12.5 & \\
\hline 0 & 0.0 & 5 & 27.8 & 5 & 10.4 & \\
\hline 30 & 100.0 & 18 & 100.0 & 48 & 100.0 & \\
\hline
\end{tabular}

Table (2) demonstrates the distribution of all study groups according to their gender. Females preponderance with female: male ratio $=1.7: 1$, but statistically there was no significant difference. $(\mathrm{P}>0.05)$. 
Table(2): distribution of study groups according to gender.

\begin{tabular}{|c|c|c|c|c|c|c|c|}
\hline \multirow{3}{*}{ Study Group } & \multicolumn{4}{|c|}{ Gender } & \multicolumn{2}{|c|}{ Total } & \multirow{3}{*}{ P value } \\
\hline & \multicolumn{2}{|c|}{ Male } & \multicolumn{2}{|c|}{ Female } & \multirow[b]{2}{*}{$\mathbf{N}$} & \multirow[b]{2}{*}{$\%$} & \\
\hline & $\mathbf{N}$ & $\%$ & $\mathbf{N}$ & $\%$ & & & \\
\hline Ulcerative Colitis & 12 & 50.0 & 18 & 42.9 & 30 & 45.5 & \multirow{4}{*}{0.855} \\
\hline Non ulcerative colitis & 6 & 25.0 & 12 & 28.6 & 18 & 27.3 & \\
\hline Control & 6 & 25.0 & 12 & 28.6 & 18 & 27.3 & \\
\hline Total & 24 & 100.0 & 42 & 100.0 & 66 & 100.0 & \\
\hline
\end{tabular}

Figure (1) describes the distribution of patients with ulcerative colitis according to the histopathological findings, in which the histological typing of ulcerative colitis grouped into chronic ulcerative colitis $26(86.67 \%)$ of cases or active on chronic ulcerative colitis $4(13.33 \%)$ cases.

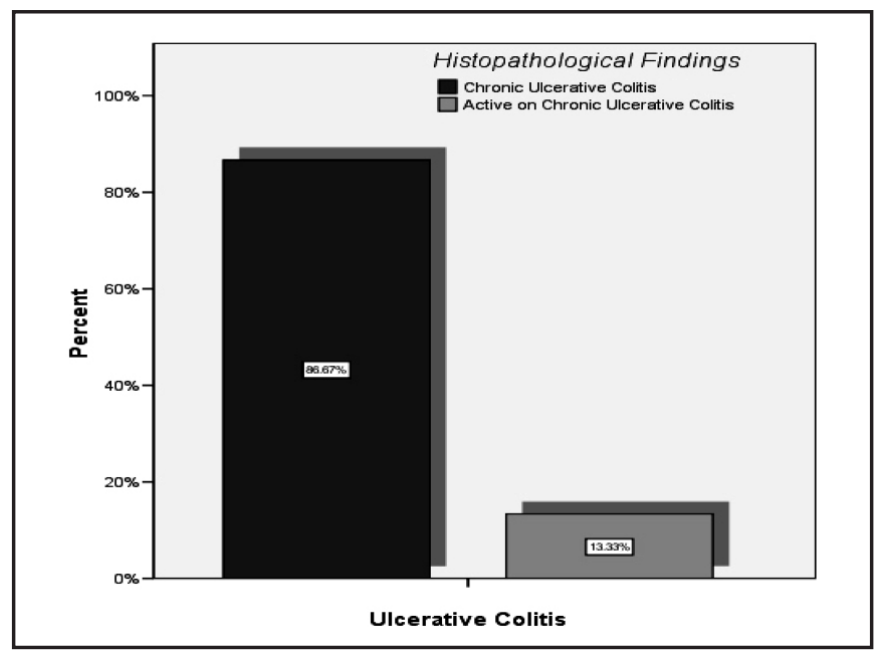

Figure(1): Distribution of patients with ulcerative colitis according to the histopathological findings.

Figure (2) describe the prevalence of E. histolytica (cyst and trophozoit) in ulcerative colitis patients by general stool examination, in which E.histolytica was positive in 21 $(70 \%)$ cases of ulcerative colitis , 16 (53.33\%) cases cyst and $5(16.67 \%)$ cases trophozoite).

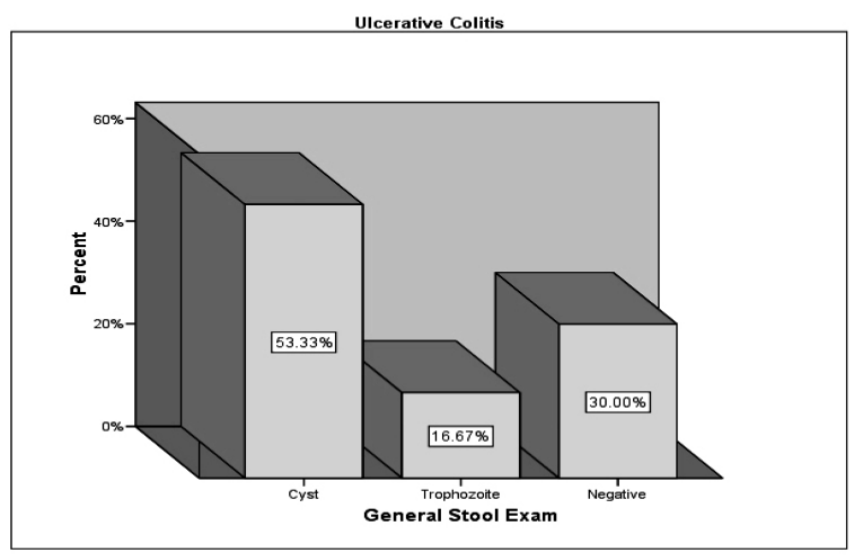

Figure (2): Prevalence of E.histolytica (cyst and trophozoit) in patients with ulcerative colitis by general stool examination.

Table (3) demonstrates the prevalence of IgG anti-E histolytica antibodies by ELISA test of all study groups, in which IgG anti E.histolytica antibodies were significantly higher in ulcerative colitis $23(76.67 \%)$ cases compared to non ulcerative colitis group, $12(66.67 \%)$ cases, so that the association between disease state (ulcerative colitis or non ulcerative colitis group) and results of ELISA test for IgG anti E.histolytica antibodies was significant compared with control group $(\mathrm{P}<0.05)$.

Table (3) Prevalence of IgG anti-E histolytica antibodies in all study groups by ELISA test.

\begin{tabular}{|c|c|c|c|c|c|c|c|c|c|}
\hline \multirow{3}{*}{$\begin{array}{l}\text { IgG anti } \\
\text { E.histolytica } \\
\text { antibodies }\end{array}$} & \multicolumn{6}{|c|}{ Study Group } & \multirow{2}{*}{\multicolumn{2}{|c|}{ Total }} & \multirow{3}{*}{$\begin{array}{c}P \\
\text { value }\end{array}$} \\
\hline & \multicolumn{2}{|c|}{$\begin{array}{c}\text { Ulcerative } \\
\text { Colitis }\end{array}$} & \multicolumn{2}{|c|}{$\begin{array}{c}\text { Non } \\
\text { ulcerative } \\
\text { colitis }\end{array}$} & \multicolumn{2}{|c|}{ Control } & & & \\
\hline & $\mathbf{N}$ & $\%$ & $\mathbf{N}$ & $\%$ & $\mathbf{N}$ & $\%$ & $\mathbf{N}$ & $\%$ & \\
\hline Positive & 23 & 76.7 & 12 & 66.7 & 7 & 38.9 & 42 & 63.6 & \\
\hline Negative & 7 & 23.3 & 6 & 33.3 & 11 & 61.1 & 24 & 36.4 & 0.030 \\
\hline Total & 30 & 100.0 & 18 & 100.0 & 18 & 100.0 & 66 & 100.0 & \\
\hline
\end{tabular}

Table (4) demonstrates the comparison between positive results for E.histolytica in both tests; ELISA and GSE in all study groups.

Concerning the detection of E. histolytica by ELISA and GSE we found that they were somewhat equal in both methods for all study groups (the ratio were about 1:1). 
Table (4): Comparison between proportions of positive results for E. histolytica in both tests; ELISA and GSE in all study groups.

\begin{tabular}{cccccc}
\hline \multirow{2}{*}{ Study Group } & \multicolumn{4}{c}{$\begin{array}{c}\text { E. histolytical IgG } \\
\text { Positive }\end{array}$} & $\begin{array}{c}\text { Positive E.histolytica } \\
\text { in GSE }\end{array}$ \\
\cline { 2 - 6 } & N & $\%$ & N & $\%$ & Ratio \\
\hline Ulcerative Colitis & 23 & 76.7 & 21 & 70 & 1.1 \\
\hline Nonulcerative colitis & 12 & 66.7 & 13 & 71.2 & 0.9 \\
\hline Control & 7 & 38.9 & 8 & 44.4 & 0.88 \\
\hline
\end{tabular}

Table (5) demonstrates the distribution of study sample according to results of GSE and IgG anti- E histolytica antibodies, We found that $75.8 \%$ of patients have positive and cyst stage; and $77.8 \%$ were have positive IgG anti-E. histolytica antibodies and trophozoit stage, while $24.2 \%$ of negative GSE results were also negative for $\mathrm{IgG}$ anti-E. histolytica antibodies, and the association was significant compared with control group $(\mathrm{P}<0.05)$.

Table (5): Distribution of study sample according to results of GSE and IgG anti- E histolytica antibodies.

\begin{tabular}{|c|c|c|c|c|c|c|c|c|c|}
\hline \multirow{3}{*}{$\begin{array}{c}\text { IgG anti- E.histolytica } \\
\text { antibodies }\end{array}$} & \multicolumn{6}{|c|}{ GSE Findings } & \multirow{2}{*}{\multicolumn{2}{|c|}{ Total }} & \multirow{2}{*}{ P value } \\
\hline & \multicolumn{2}{|c|}{ Cyst } & \multicolumn{2}{|c|}{ Trophozoite } & \multicolumn{2}{|c|}{ Negative } & & & \\
\hline & $\mathbf{N}$ & $\%$ & $\mathbf{N}$ & $\%$ & $\mathbf{N}$ & $\%$ & $\mathbf{N}$ & $\%$ & \multirow{4}{*}{0.019} \\
\hline Positive & 25 & 75.8 & 7 & 77.8 & 10 & 41.7 & 42 & 63.6 & \\
\hline Negative & 8 & 24.2 & 2 & 22.2 & 14 & 58.3 & 24 & 36.4 & \\
\hline Total & 33 & 100.0 & 9 & 100.0 & 24 & 100.0 & 66 & 100.0 & \\
\hline
\end{tabular}

\section{Discussion:}

This case - series study included 48 patients with colitis attended the Gastroenterology and Hepatology Teaching Hospital (a major referral center in Baghdad) (45.45\% were ulcerative colitis and $27.27 \%$ were non ulcerative colitis group) and 18 healthy individuals as control (27.27\%). The peak age incidence of patients with ulcerative colitis in this study group was between $21-50$ years (mean=33.11 years \pm 15.682); and females preponderance with female: male ratio $=1.7: 1$; similar result was reported by an Iraqi study Shafeek (2002). (11). In other study done by Al-akyshee (2002) the peak age incidence of ulcerative colitis was between 2030 years with a mean of 35.5 years and female: male ratio 1.2:1 (12) Studies from united state of America describe two peak age incidence for ulcerative colitis patients one between 20-40 years and one after 70 years, with an equal sex incidence or slightly female preponderance may be due to hormons. $(13,14)$ In this study and other Iraqi studies no second peak had been identified because of low number of patients after age of 70 years. The use of colonoscopy in detection of mucosal disease, its severity and extent is well established and in diagnosis of ulcerative colitis versus other cause of diarrhea with blood, generally showed good correlation with histological typing. (15)However a minor discrepancy and under estimation of endoscopic finding in comparison to histology is well recognize in comparison to literature. There for, it seems appropriate to recommend use of both endoscopy and histology for assessment of disease activity and extent. The colonoscopy with multiple biopsies has come to be the recommended routine investigation for diagnosis of ulcerative colitis versus other cause of diarrhea with blood like infected colitis patients. (16) Still colonoscopy is not recommended as a routine diagnostic approach in intestinal amoebic ulcerations because it may increase the likelihood the perforation during instillation of air to expand the colon. (17) Histological evaluation has been shows to be more sensitive tool than endoscopy alone for determination of disease activity. (19) Moum et al (1999) have found that overall agreement between endoscopic and histological findings regarding extent of disease to be $78 \%$ at time of diagnosis compared to be $60 \%$ at time of follow up. (20) In this study anti IgGE.histolytica antibodies were significantly positive in ulcerative colitis patients $(76.67 \%)$ compared to control group (38.9\%); and anti IgG E.histolytica antibodies were significantly positive in non ulcerative colitis group $(66.67 \%)$ compared to control $(38.9 \%),(\mathrm{P}<0.05)$. In other studies up to $25 \%$ of uninfected individuals in endemic areas have anti-amoebic 
antibodies due to previous, often undiagnosed infection with E. histolytica. (21) A recent study in Turkey showed that amoeba infection in patients with IBD, especially those with $\mathrm{UC}$, is more prevalent than in the normal population (22), Bayramicli et al (23) explored the presence of E.histolytica in $69 \%$ of the cases of ulcerative colitis patients. While In a study carried out in the Province of Antalya determined the rate of amebiasis in $43 \%$ of patients with ulcerative colitis. (24) These resultes are in agreement with our study results. Other studies showed a variable incidence of E. histolytica in patients with ulcerative colitis ranging from low result (4.85\%) in a study done by Prokopowicz et al (25) to a higher incidence like that detected by Suleymanlar et al that found E. histolytica cysts and trophozoites in 22 (54 $\%$ ) of the patients with ulcerative colitis patients.(26) This difference in incidence of E.histolytica in patients with ulcerative colitis is may be due to environmental factors, high temperature, humidity, and lower immune resistance against infection, in addition to poor hygiene.(26), so in Iraq there is high incidence of E.histolytica in patients with ulcerative colitis. E.histolytica was positive by general stool examination in $70 \%$ of cases with ulcerative colitis (53.33\% as cyst and $16.67 \%$ as trophozoit); this concomitance infection is important to know because E.histolytica should be properly treated before starting steroid therapy in patient with ulcerative colitis. (27) General stool examination for E.histolytica was parallel to the positive result obtained by ELISA for IgG anti E.histolytica antibodies (76.67\%) Therefore, we believe that E.histolytica must be explored in the feces before planning a diagnostic scheme for cases diagnosed as IBD. (28)However other studies considered the stool microscopy as a relatively poor method for diagnosing intestinal amoebiasis because it is timeconsuming, laborious, and requires specific expertise. (29) The high percentage of anti E.histolytica antibodies in patients with non ulcerative colitis group may be the cause of there disease and in need to be followed with their physician to confirm the diagnosis and get the exact incidence of E.histolytica among the patients with chronic diarrhea with blood.

\section{Conclusion:}

IgG anti E.histolytica Abs were significantly higher in ulcerative colitis $(76.67 \%)$ compared to non ulcerative colitis group (66.67\%). There was a close association between E.histolytica and ulcerative colitis also there is a high incidence of E.histolytica among group of non ulcerative colitis patients \& sensitivity of detection of E.histolytica by ELISA and GSE is rather equal.

\section{Author's contributions:}

Dr Kifah Hamdan : collection of cases and histopathological study

Dr Maher Mahmoud collection of data and statistical analysis

Dr Luay Ibraheam Serological and microbiological study of the cases

References:

1- Auerbach M. ulcerative colitis an inflammatory bowel disease 2008:www.cigna.com.2- David J. Maron. Ulcerativecolitis.American society of colon and rectal surgeon 2008; www.Fascrs.org/.

3- American Gastroenterology Association. The burden of gastrointestinal disease.Chapter 4. Intestinal diseases. 2014:30-5.

4- Jewell DP .: Feldman M. Sleisenger-MH, Schwarchmidt BF et al. (eds.), Ulcerative colitis .Text book of gastrointestinal and liver disease 6 ed. W.B. - Saunders company, 2014; 1735.

6- Clickman RM. Inflammatory Bowel Disease (ulcerative colitis and crohns disease). In : Fauc, Braunwaid et al (eds.) , Harrison's principle of internal medicine, 16th ed., MaGraw Hill 2014 ; 1777

7-Lotus EV, Jr. Clinical epidemiology of inflammatory bowel disease: Incidence, prevalence, and environmental influences. Gastroenterology 2004; 126:1504-1517.

8- Timmer A. Environmental influences on inflammatory bowel disease manifestation. Digestive Disease 2009; 21:91-104

9- American Gastroenterological Association. Position statement:

Guidelines on osteoporosis in gastrointestinal diseases Gastroenterology 2010:124:791-4.

10- Sartor RB. Cytokines in intestinal inflammation: patho-physiological and clinical considerations. Gastroenterology.1994; 106: 533-539.

11-Shafeek M, Al-fayadh MH. Comparesion Study Between Endoscopic and Histological Finding in Ulcerative Colitis patients in IRAQ.(Accepted thesis submitted to the Iraqi Commission for medical specialization of gastroenterology 2002).

12- Al-Akayshee R, Shubbar A; Chronic colitis Clinical, Endoscopic, and Histopathological Evaluation in Iraqi patients.(Iraqi Journal of gastroenterology (IJGE); volum 1; issue 1; October 2001; page 11-17).

13- John S, Groff MD. Colitis; radiation, microscopic, collagenous and pseudomembranous; McNally P.R.; Gil liver secrets; Philadelphia, 1-Ianiy and I3elens Inc 1997; 
$344-$

14- Bernstein C, Katz S. Guidelines for osteoporosis and inflammatory bowel disease. A guide to diagnosis and management for the gastroenterologist (monograph). The American College of Gastroenterology, 2003.

15- Geboes K., Riddell R. ,ost A. et al. A reproducible grading scale for histological assessment of inflammation in ulcerative colitis. Gut, 2000;

47 : 404-409.

16- Thomas T, Abrams KA, Robinson RJ, et al. Metaanalysis: cancer risk of low-grade dysplasia in chronic ulcerative colitis. Aliment PharmacolTher 2007; 25:657668

17- Eaden JA, Abrams KR, Mayberry JF. The risk of colorectal cancer in ulcerative colitis: a meta-analysis. Gut 2001; 48:526-535

18-RosaiJ.Akarman»ssurgicalpathology. GITpathology.6th ed. Mosby Co.2008.

19- Vinod K. Dhawan. Amebiasis. American society of microbiology conference.2008; Aug.

20- Moum B, Ekloom A, Morten H.V. et al . Change in the extent of colonoscopiic and Histological Involvment in ulcerative colitis oveer time .A J G, 1999;6:1564-69.

21- Stanley SL Jr. Amebiasis. Lancet 2003 Mar 22; 361(9362): 1025-34.

22- SebnemUstun, HandeDagci, UmitAksoy, YukselGuruz, GalipErsoz. Prevalence of amebiasis in inflammatory bowel disease in Turkey. World J Gastroenterol 2003; 9(8):18341835

23- Patel AS, DeRidder PH. Amoebic colitis masquerading as acute inflammatory bowel disease: the role of serology in its diagnosis. J ClinGastroenterol 1989; 11(4):407-410.

24- AddibO,ZiglamH,Conlongpg,Invasiveameobisis complicating inflammatory bowel diseas;Libyanjornal.of medicine .2007; sept:(1819-1821).

25- Prokopowicz D, Zagorski K, Kramarz P. Amoebiasis-a problem in patients with ulcerative colitis. WiadLek 1994;

47: $248-251$

26- Süleymanlar I, Atilgan S, Ertugrul C, Isitan F. Ulseratifkolitve intestinal amebiasisbirlikteligivetedavide karsilasilansorunlar. Gastroenterology, 1996; 7:22.

27- Underwood JC, 2004. [General and Systematic Pathology], 4th ed. Churchill Livingstone.pp: 370- 374.

28- Baily \&Scott, 2009, $13 t$ ed. Diagnostic microbiology Mosby

29- Ustun S, Dagci H, Aksoy U, Guruz Y, Erroz G, Prevalence of amebiasis in inflammatory bowel disease in Turkey, World Journal of gastroenterology 2003 August ; 9(8):1834-1835. 